TRANSACTIONS OF THE

AMERICAN MATHEMATICAL SOCIETY

Volume 186, December 1973

\title{
ON THE STRUCTURE OF SEMIGROUPS WHICH ARE UNIONS OF GROUPS $\left({ }^{1}\right)$
}

BY

\section{R. J. WARNE}

ABSTRACT. We characterize semigroups $S$ which are unions of groups as generalized Schreier products of groups, semilattices of right zero semigroups, and semilattices of left zero semigroups. We then give several specializations of this result utilizing Schreier products, semidirect products, and direct products.

In a well-known theorem ([2], [3, Theorem 4.6]), Clifford characterizes semigroups $S$ which are unions of groups (more precisely, unions of their subgroups) as associative (2) semilattices $Y$ of completely simple semigroups $\left(S_{y}: y \in Y\right)$, i.e. $S=U\left(S_{y}: y \in Y\right.$, a semilattice), the $S_{y}$ are pairwise disjoint completely simple semigroups, and $S_{y} S_{t} \subseteq S_{y \wedge t^{*}}$ Hence, for each $y \in Y, S_{y}=I_{y} \times J_{y}$ (Car: tesian product) where $I_{y}$ is a left group and $J_{y}$ is a right zero semigroup. We define a product on $I=\bigcup(I, y \in Y)$ so that $I$ becomes a lower associative semilattice $Y$ of left groups $\left(I_{y}: y \in Y\right)\left(a \in I_{y}, b \in I_{z}, c \in I_{w}\right.$, and $y \geq z \geq w$ imply $\left.a(b c)=(a b)_{c}\right)$ and we define a product on $J=\bigcup\left(J_{y}: y \in Y\right)$ so that $J$ becomes a semilattice $Y$ of right zero semigroups $\left(J_{y}: y \in Y\right)$. In $\$ 1$, we characterize $S=\bigcup\left(I_{s} \times J_{s}: s \in Y\right)$ as a generalized Schreier product of $I$ by $J$-more precisely, the subsemigroup $U\left(I_{y} \times J_{y}: y \in Y\right)$ of this product ("generalized" as the second conrdinates multiply as a semidirect product instead of a direct product). We then give a similar characterization of $I$ by means of groups and semilattices of left zero semigroups. In $\$ 2$, we characterize a band (noncommutative semilattice) of maximal left groups, $S$, as a Schreier product of $I$ by associative $J$. In $\$ 3$, we characterize orthodox $S(E(S)$, the set of idempotents of $S$, is a semigroup) as a generalized semidirect product of orthodox $I$ by upper associative $J$. Specialization of this characterization to idempotent semigroups yields a variant of [10, Theorem 2]. In $\S 4$, we characterize generalized $R$-unipotent $S(e, f \in E(S)$ and $e f=f$ imply $f e g f g=f g$ for all $g \in E(S))$ as a semidirect

Received by the editors August 11, 1972.

AMS (MOS) subject classifications (1970). Primary $20 \mathrm{M} 10$.

Key words and phrases. Union of groups, Schreier product, semidirect product, spined product, semilattice of right zero semigroups.

(1) A summary of this paper appeared as a research announcement in the Semigroup Forum 5 (1973), 323-330.

(2) We differ from the usual terminology $[3]$ in that we do not require a semilattice of semigroups to obey associativity.

Copyright $\odot$ 1974, American Mathematical Society 
product of upper associative $J$ by associative $I$. In $\$ 5$, we characterize an orthodox band of maximal left groups, $S$, as a semidirect product of $I$ by associative $J$. Specialization of this characterization to bands of maximal left zero semigroups yields a variant of [10, Theorem 3]. In $\$ 6$, we characterize a generalized $R$-unipotent band of maximal left groups, $S$, as a direct product of associative $I$ and associative $J$. Upon specializing this characterization to idempotent semigroups, we obtain a variant of a result of Kimura [5, Theorem 2]. (In this case, Kimura terms $S$ a spined product of $I$ and $J$.) In $\$ 7$, we characterize -unipo- $^{-}$ tent $S$ (each principal left ideal of $S$ contains a unique idempotent generator or equivalently $S$ is an associative semilattice $Y$ of right groups $\left(S_{y}: y \in Y\right)$ ) as a semidirect product of associative $J$ by associative $I$ such that $I_{y}^{y}$ is a group for each $y \in Y$ (Theorem 7.2). Upon specializing Theorem 7.2 to bands of groups, we obtain a result of Bailes [1, Theorem 30]. The dual of Theorem 7.2 together with Clifford's characterization of associative semilattice of groups ([2], [3, Theorem 4.11]) may be applied to further refine the structure theorems in $\$ \$ 4$ and 6 .

An excellent account of the previous structure theory for orthodox $S$, especially of the Fantham, Preston, and Yamada theorems, is given in [4]. We also refer the reader to [4] for a bibliography of this theory.

1. Semigroups which are unions of groups. Let $S$ be a semigroup which is a union of groups. We show $S=\bigcup\left(I_{y} \times J_{y}: y \in Y\right)$ under the multiplication $(A, a)(B, b)=\left(A \circ B^{a} \circ b^{\left(a^{B}\right)}, a^{B} * b\right)$ where "o" denotes the multiplication on $I=\bigcup\left(I_{y}: y \in Y\right)$; " $*$ " de notes the multiplication on $J=\bigcup\left(J_{y}: y \in Y\right)$; for $a, b$ $\epsilon J, b^{a} \in H_{y}$, some fixed maximal subgroup of $I_{y}$; and, for $B \in I$ and $a \in J, B^{a}$ $\epsilon I$ and $a^{B} \in J$. The $B^{a}$ and $b^{a}$ obey conditions similar to those given in a Schreier product while if we set $a^{B}=a \theta_{B}$, then $B \rightarrow \theta_{B}$ is a (partial) homomorphism of $(I, \circ)$ into $T_{J}$, the full transformation semigroup on $J$ and $\theta_{B}$ is something similar to an endomorphism of $(J, *)$. We say "similar" as some mixing occurs as indicated in the product given above. Another condition involves associativity in $\mathrm{J}$.

Let $T$ be an upper associative semilattice $Y$ of right groups $\left(T_{y}: y \in Y\right)$. We obtain the structure of $T$ by letting $H_{y}=I_{y}$ in the structure theorem for $S$. Some modifications arise due to the absence of full associativity in $T$. The dual of this result will further refine the structure of $S$. We conclude this section by considering the case where $E(T)$ is partial subgroupoid of $T$.

Before beginning our program, we will need several definitions and some preliminary discussion. Unless we specify to the contrary, we will adopt the terminology of Clifford and Preston [3].

First, we note that the concepts of "semilattice" and "commutative idempotent semigroup" are equivalent. We just set e $\wedge f=e f$ and vice versa [3, 
Theorem 1.12]. A groupoid $S$ which is a union of a collection of pairwise disjoint subgroupoids $\left(S_{y}: y \in Y\right)$ where $Y$ is a semilattice and $S_{y} S_{t} \subseteq S_{y t}$ for all $y, t \in$ $Y$ is termed a semilattice $Y$ of groupoids $\left(S_{y}: y \in Y\right)$. If $a \in S_{u}$ and $b \in S_{v}$, we say $a \leq b$ if and only if $u \leq v$. If $(a b) c=a(b c)$ for $a \geq b \geq c(a \leq b \leq c), S$ is termed a lower (upper) associative semilattice $Y$ of groupoids $\left(S_{y}: y \in Y\right)$. If $S$ is a semigroup, $S$ is termed an associative semilattice $Y$ of semigroups $\left(S_{y}: y\right.$ $\epsilon Y)$.

Let $R, \mathcal{L}, \mathcal{H}$, and $\mathcal{D}$ denote Green's relations [3] on a semigroup $S$, i.e. $R=$ $\left((a, b) \in S^{2}: a \cup a S=b \cup b S\right), \mathcal{H}=\mathbb{R} \cap \mathscr{L}$, and $\mathfrak{D}=\mathscr{R} \circ \mathscr{L}=\left((a, b) \in S^{2}:(a, x) \epsilon\right.$ $\mathscr{R}$ and $(x, b) \in \mathscr{L}$ for some $x \in S)$.

Let $S$ be a semigroup which is a union of groups. By Clifford's theorem ([2], [3, Theorem 4.6]), $S$ is an associative semilattice $Y$ of completely simple semigroups $\left(S_{y}: y \in Y\right)$. By the Rees theorem ([7], [3, Theorem 3.5]), $S_{y}=G_{y} \times M_{y} \times$ $N_{y}$ (set direct product) where $G_{y}$ is a group and $M_{y}$ and $N_{y}$ are sets with $M_{y} \cap$ $N_{y}^{y}=\left(1_{y}\right)$, a one element set. Furthermore, if $(g, i, j),(b, p, q) \in S_{y},(g, i, j)$. $(b, p, q)=\left(g f_{y}(j, p) b, i, q\right)$ where $(j, p) \rightarrow f_{y}(j, p)$ is a mapping of $N_{y} \times M_{y}$ into $G_{y^{*}}$ By a remark of Clifford [3, p. 95], we may take $f_{y}\left(1_{y}, i\right)=f_{y}\left(j, 1_{y}\right)=e_{y}$ for all $j \in N_{y}, i \in M_{y}$ where $e_{y}$ is the identity of $G_{y}$. We will let $e_{y}=\left(e_{y}, 1_{y}, 1_{y}\right)$. If $S$ is orthodox, $\left(f_{y}(j, i), 1_{y}, 1_{y}\right)=\left(e_{y}, 1_{y}, j\right)\left(e_{y}, i, 1_{y}\right) \in E\left(S_{y}\right)$. Hence $f_{y}(j, i)=$ $e_{y}$ for all $i \in M_{y}$ and $j \in N_{y}$. The facts and notation of this paragraph will be used without explicit mention. The given multiplication in $S$ will be denoted by juxtaposition.

Our first structure theorem will be the consequence of eleven lemmas.

Let $I_{y}=G_{y} \times M_{y} \times\left(1_{y}\right), J_{y}^{*}=G_{y} \times\left(1_{y}\right) \times N_{y}$, and $J_{y}=\left(e_{y}\right) \times\left(1_{y}\right) \times N_{y^{*}}$ Let $I=\bigcup^{\prime}\left(I_{y}: y \in Y\right)$ and $J=\bigcup_{y}\left(J_{y}: y \in Y\right)$. In the remainder of this paper, the elements of $I(J)$ will be denoted by capital (lower case) italic letters.

Lemma 1.1. For each $y \in Y, I_{y}$ is a left group. If $A \in I_{y^{,}} B \in I_{z^{\prime}}$ and $z \leq$ $y, A B \in I_{z}$.

Proof. By [3, Theorem 1.27], $I_{y}$ is a left group. Let $A=\left(g, i, 1_{y}\right) \in I_{y}$, $B=\left(b, j, 1_{z}\right) \in I_{z}$ and $y \geq z$. Hence, $A B=(v, k, s) \in S_{z}$, say. Thus, $(v, k, s)$. $\left(e_{z}, 1_{z}, 1_{z}\right)=A\left(h, j, 1_{z}\right)\left(e_{z}, 1_{z}, 1_{z}\right)=A B=(v, k, s)$. Hence, $A B \in I_{z^{*}}$

Lemma 1.2. For each $y \in Y, J_{y}$ is a right zero semigroup. If $a \in J_{y}, b \in$ $J_{t}$, and $y \leq t, a b \in J_{y}^{*}$.

Proof. By a simple calculation $J_{y}$ is a right zero semigroup. Suppose that $a=\left(e_{u}, 1_{u}, s\right) \in J_{u}, b=\left(e_{v}, 1_{v}, t\right) \in J_{v}$, and $u \leq v$. Hence, $a b=(z, p, q) \in S_{u}$, say. Thus, $\left(e_{u}, 1_{u}, s\right)(z, p, q)=a(a b)=a b=(z, p, q)$ and, hence, $a b \in J_{u}^{*}$. 
Lemma 1.3. Every element of $S$ may be uniquely expressed in the form $x=$ Aa where $A \in I_{y}$ and $a \in J_{y}$ for some $y \in Y$.

Proof. If $x=(g, i, j) \in S_{y}, x=\left(g, i, 1_{y}\right)\left(e_{y}, 1_{y}, j\right)$.

Lemma 1.4. For eacb $a \in J_{u}$ and $B \in I_{v}(u, v \in Y)$, there exist a unique element $B^{a} \in I_{u v}$ and a unique element $a^{B} \in J_{u v}$ such that $a B=B^{a} a^{B}$. Furthermore $a B \Re B^{a}$ and $a B \mathscr{L} a^{B}$.

Proof. Let $a \in J_{y}$ and $B \in I_{t^{*}}$ Thus, $a B \in S_{y t^{*}}$ Hence, by Lemma 1.3, there exists a unique $u \in I_{y t}^{y}$ and $v \in J_{y t}^{t}$ such that $a B^{y t} u v$. Let $u=B^{a}$ and $v=a^{B}$. The last statement is valid by a simple calculation.

If $A \in I_{s}$ and $B \in I_{t}$, define $A \circ B=A\left(B^{e} s\right)$.

Lemma 1.5. $(I, 0)$ is a lower associative semilattice $Y$ of left groups $\left(I_{y}\right.$ : $y \in Y$ ). If $A \geq B \in I, A \circ B=A B$.

Proof. By Lemmas 1.4 and $1.1, I_{u}^{\circ} I_{v} \subseteq I_{u v}$. Next, let $A \in I_{s}, B \in I_{t}$ and $s \geq t$. Thus, $e_{s} B \in I_{t}$ by Lemma 1.1 and, hence, $e_{s} B=\left(e_{s} B\right) e_{t}$. However, $e_{s} B$ $=B^{e} s_{e}^{B}$ by Lemma 1.4. Hence, $B^{e}=e_{s} B$ by Lemma 1.4 and Lemma 1.3. Thus, $A \circ B=A\left(B^{e} s\right)=A\left(e_{s} B\right)=\left(A e_{s}\right) B=A B$. Hence, if $C \leq B$ in $I, A \circ(B \circ C)=A$ $\circ(B C)=A(B C)=A(B C)=A \circ(B \circ C)$. Q.E.D.

If $a=\left(z, 1_{u}, r\right) \in J_{u}^{*}$, define $a^{\prime}=\left(e_{u}, 1_{u}, r\right) \in J_{u}$. In other words, $a^{\prime}$ is the identity of the maximal subgroup of $S$ containing $a$. If $x \in J_{u}$ and $y \in J_{v}$, define $x * y=\left(x^{e} v_{y}\right)^{\prime}$ (here, $e_{v}$ is considered to be an element of $I_{v}$ ).

Lemma 1.6. $(J, *)$ is a semilattice $Y$ of right zero semigroups $\left(J_{y}: y \in Y\right)$. If $a \leq b$ in $J, a * b=(a b)^{\prime}$.

Proof. By Lemmas 1.2 and 1.4, $J_{s} * J_{t} \subseteq J_{s t^{\circ}}$ Next, let $a \in J_{u^{\prime}} b \in J_{v}$, and $u \leq v$. Since $\left(a^{e} v, a e\right) \in \mathfrak{L}$ by Lemma $1.4,\left(a^{e} v b, a e v b\right) \in \mathfrak{L}$ and hence $\left(a^{e} v b\right.$, $a b) \in \mathscr{L}$. However, $a^{e} v b, a b \in J_{u}^{*}$ by Lemma 1.2. Hence, $(a b)^{\prime}=\left(a^{e} v b\right)^{\prime}=a * b$. Q.E.D.

If $r \in J_{u^{\prime}}, s \in J_{v}$, and $u \leq v$, define $s^{r}=r s e_{u}$. Hence, if $r s=\left(z, 1_{u}, j\right) \epsilon$ $J_{u}^{*}$ (Lemma 1.2), $s^{r}=\left(z, 1_{u}, 1_{u}\right)$. We note that $s^{r}$ is contained in the maximal subgroup with identity $e_{u} \cdot$

Lemma 1.7. If $s \leq t \in J$, $s t=t^{s}(s * t)$.

Proof. Let $s \in J_{u}, t \in J_{v}$, and $u \leq v$. By Lemma 1.2, st $=\left(z, 1_{u}, k\right) \in J_{u}^{*}$, say. Hence, $s t=t^{s}(s t)^{\prime}$. Hence, by Lemma $1.6, s t=t^{s}(s * t)$.

Lemma 1.8. If $A \geq B \in I, c \in J$,

(a) $c^{A \circ B}=\left(c^{A}\right)^{B}$,

(b) $(A \circ B)^{c}=A^{c} \circ B^{\left(c^{A}\right)}$. 
Proof. Utilizing Lemmas 1.1 and 1.4, $c(A B)=(A B)^{c} c^{A B}$ while $(c A) B=$ $\left(A^{c} c^{A}\right) B=A^{c}\left(c^{A} B\right)=A^{c} B^{\left(c^{A}\right)}\left(c^{A}\right)^{B}$. Thus, we apply Lemmas 1.4, 1.5, and 1.1.

Lemma 1.9. If $a \leq b \in J, C \in I$, and $b=a^{\left(C^{b}\right)}$,

(a) $(a * b)^{C}=b * b^{C}$,

(b) $b^{a} \circ C^{a * b}=\left(C^{b}\right)^{a} \circ\left(b^{C}\right)^{(b)}$.

Proof. We now proceed as in the proof of Lemma 1.8. Utilizing Lemmas 1.7 and 1.6, $(a b) C=\left(b^{a}(a * b)\right) C=b^{a}((a * b) C)=b^{a}\left(C^{a * b}(a * b)^{C}\right)=\left(b^{a} C^{a * b}\right)(a * b)^{C}$ while $a(b C)=a\left(C^{b} b^{C}\right)=\left(a C^{b}\right) b^{C}=\left(C^{b}\right)^{a}\left(b b^{C}\right)=\left(C^{b}\right)^{a}\left(b^{C}\right)^{(b)}\left(b * b^{C}\right)$. Thus, we apply Lemmas $1.4,1.5,1.6$, and 1.1 .

Lemma 1.10. If $a \leq b \leq c \in J$ and $k=a^{\left(c^{b}\right)}$,

(a) $b^{a} \circ c^{a * b}=\left(c^{b}\right)^{a} \circ(b * c)^{(k)}$,

(b) $(a * b) * c=k *(b * c)$.

Proof. Utilizing Lemma 1.7, $a(b c)=a\left(c^{b}(b * c)\right)=\left(a c^{b}\right)(b * c)=\left(c^{b}\right)^{a} k(b * c)=$ $\left(c^{b}\right)^{a}(b * c)^{(k)} k *(b * c)$ while $(a b) c=\left(b^{a}(a * b)\right) c=b^{a}((a * b) c)=b^{a} c^{a * b}(a * b) * c$.

Lemma 1.11. $S \cong U\left(I_{y} \times J_{y}: y \in Y\right)$ under the multiplication

$$
(A, a)(B, b)=\left(A \circ B^{a} \circ b^{\left(a^{B}\right)}, a^{B} * b\right)
$$

Proof. Using Lemmas 1.5 and 1.7, $(\bar{A} a)(B b)=A(a B) b=\left(A B^{a}\right)\left(a^{B} b\right)=$ $\left(A \circ B^{a} \circ b^{\left(a^{B}\right)}\right)\left(a^{B} * b\right)$. Thus, using Lemmas 1.3, 1.5 and 1.6, $(A a) \phi=(A, a)$ is the required isomorphism.

We are now in a position to give our structure theorem for semigroups which are unions of groups.

Let $(I, 0)$ be a lower associative semilattice $Y$ of left groups, $\left(I_{y}: y \in Y\right)$; for each $y \in Y$, let $H_{y}$ be a fixed subgroup of $I_{y}$; let $(J, *)$ be a semilattice $Y$ of right zero semigroups $\left(J_{y}: y \in Y\right)$. For each $a \in J_{u}$ and $B \in I_{v}(u, v \in Y)$, let $B^{a} \in I_{u v}$ and $a^{B} \in J_{u v^{*}}$. For each $a \in J_{u}$ and $b \in J_{v}$ with $u \leq v$ in $Y$, let $b^{a} \in H_{u}$. Assume the conditions:

1. If $A \geq B \in I, c \in J$,

(a) $c^{A \circ B}=\left(c^{A}\right)^{B}$,

(b) $(A \circ B)^{c}=A^{c} \circ B^{\left(c^{A}\right)}$.

2. If $a \leq b \in J, C \in I$, and $b=a^{\left(C^{b}\right)}$,

(a) $(a * b)^{C}=b * b^{C}$,

(b) $b^{a} \circ C^{a * b}=\left(C^{b}\right)^{a} \circ\left(b^{C}\right)^{(b)}$.

3. If $a \leq b \leq c \in J$, and $k=a^{\left(c^{b}\right)}$,

(a) $b^{a} \circ c^{a * b}=\left(c^{b}\right)^{a} \circ(b * c)^{(k)}$,

(b) $(a * b) * c=k *(b * c)$. 
We term $U\left(I_{y} \times J_{y}: y \in Y\right)$ under the multiplication

4. $(A, a)(B, b)=\left(A \circ B^{a} \circ b^{\left(a^{B}\right)}, a^{B} * b\right)$, a ge neralized Schreier product of I by $J$.

Theorem 1.12. A semigroup $S$ is a union of groups if and only if $S$ is a generalized Schreier product of $I$ by $J$ for some $I$ and $J$.

Proof. Let $S$ be a semigroup which is a union of groups. Hence, $S$ is a generalized Schreier product of $I$ by $J$ by Lemmas $1.5,1.6$, the definition of $b^{a}$ $(a, b \in J)$, and Lemmas $1.4,1.8,1.9,1.10$, and 1.11 .

Conversely, let $S$ be a generalized Schreier product of $I$ by $J$. Let us establish associativity. If $(A, a) \in S$, let $(A, a)_{1}=A$ and $(A, a)_{2}=a$. Hence, if $(A, a),(B, b)$, and $(C, c) \in S$, and $b=\left(a^{B}\right)^{\left(C^{b}\right)}$ and $k=c^{\left(b^{C}\right)}$,

$$
\begin{aligned}
(((A, a)(B, b))(C, c))_{1} & =\left(A \circ B^{a} \circ\left(b^{a}{ }^{B} \circ C^{a^{B} * b}\right) \circ c^{\left(\left(a^{B} * b\right)^{C}\right)}\right. \\
& \left.\left.=A \circ B^{a} \circ\left(C^{b}\right)^{a^{B}} \circ\left(b^{C}\right)^{(b)} \circ c^{(b * b}\right)^{C}\right) \text { by 2(b) and 2(a) } \\
& \left.\left.=A \circ B^{a} \circ\left(C^{b}\right)^{\left(a^{B}\right)} \circ(k)^{(b)} \circ\left(b^{C} * c\right)^{((b)}\right)^{(k)}\right) \text { by 3(a) } \\
& =A \circ\left(B \circ C^{b} \circ k\right)^{a} \circ\left(b^{C} * c\right)^{\left(a^{\left(B \circ C^{b} \circ k\right)}\right)} \text { by } 1(b) \text { and } 1(a) \\
& =((A, a)((B, b)(C, c)))_{1}
\end{aligned}
$$

while

$$
\begin{aligned}
(((A, a)(B, b))(C, c))_{2} & =\left(a^{B} * b\right)^{C} * c \\
& =\left(b * b^{C}\right) * c \text { by } 2(a) \\
& =(b)^{(k)} *\left(b^{C} * c\right) \text { by } 3(b) \\
& =((A, a)((B, b)(C, c)))_{2} \text { by } 1(a) .
\end{aligned}
$$

Hence, $((A, a)(B, b))(C, c)=(A, a)((B, b)(C, c))$.

Let $T_{s}=I_{s} \times J_{s}$ for $s \in Y$. For each $j \in J_{s}$, let $T_{(s, j)}=I_{s} \times(j)$. Using (4) and the fact $\left(I_{s}, 0\right)$ is a left simple (right cancellative semigroup) a simple calculation shows that $T_{(s, j)}$ under (4) is a left simple (right cancellative) semigroup. Hence, $T(s, j)$ is a left group for each $j \in J_{s}$. Furthermore, $T(s, j) T(s, k)$ $\subseteq T_{(s, k)}$. Let $a, b \in T_{s}$, i.e. $a \in T_{(s, j)}$ and $b \in T_{(s, k)}$, say. Thus, since $a b$ $\epsilon T_{(s, k)}$, there exists $y \in T_{(s, k)}$ such that $y a b=b$. Thus, $T_{s}$ is a simple semigroup. Next, suppose that $e, f \in E\left(T_{s}\right)$ and $e f=f e=f$. Hence, $e, f \in T(s, j)$, for some $j \in J_{s}$, and, thus, $e=f$, since $E\left(T_{(s, j)}\right)$ is a left zero semigroup. 
Hence, $T_{S}$ is a completely simple semigroup. Thus, $S$ is a union of completely simple semigroups, and, hence $S$ is a union of groups by a theorem of Clifford [3, Theorem 4.6].

Notation. Henceforth, if $A, B \in I_{u}$ and $c, d \in J_{u}$, we will write $A \sim B \sim c$ $\sim d$ while if $r \in J_{v}$ with $v \leq u$, we will write $r \leq A$.

In the statement of Theorem 1.12 , the only products in $(I, \circ)$ used are $A \circ B$ $(A \geq B)$. We will describe these products as a generalized Schreier product of a group by a semilattice of left zero semigroups (to save space, we will state the dual result).

Make the following modifications in the statement of Theorem 1.12. Let $I_{y}$ $=H_{y}$ and $H=\bigcup\left(H_{y}: y \in Y\right.$ ) (no further restriction on $H$ ). If $B \sim a \sim b(B \in I$; $\left.a, b^{y} \in J\right), B^{a}=B$ and $b^{a} \in E(H)$. In (1), $A \sim B \geq c$ while in (2), $C \geq b$. Assuming these modified conditions, we term a groupoid $\bigcup\left(H_{y} \times J_{y}: y \in Y\right)$ under the multiplication,

$$
(A, a)(B, b)= \begin{cases}\left(A \circ B^{a} \circ b^{\left(a^{B}\right)}, a^{B} * b\right) & \text { if } a \leq b, \\ \text { some element } \in H_{s t} \times J_{s t} & \text { if } a \$ b \quad\left(a \in J_{s}, b \in J_{t}\right),\end{cases}
$$

an upper generalized Schreier product of $H$ by $J$. (We note $A, B^{a}, b^{\left(a^{B}\right)} \in H_{s}$ )

Let $U$ and $V$ be upper associative semilattices of semigroups. We say $U$ is upper isomorphic to $V$ if there exists a bijection $\theta$ of $U$ onto $V$ such that $r, s \in U$ and $r \leq s$ (in $U$ ) imply $(r s) \theta=r \theta s \theta$. The dual of the following theorem further refines the structure of $(I, o)$ in Theorem 1.12 .

Theorem 1.13. $S$ is an upper associative semilattice of right groups if and only if $S$ is upper isomorphic to an upper generalized Schreier product of $H$ by $J$ for some $H$ and $J$.

Proof. This proof parallels that of Theorem 1.12 with slight modifications which we now indicate. Utilizing [3, Theorem 1.27], let $I_{y}=G_{y} \times\left(1_{y}\right)$ and $J_{y}$ $=\left(e_{y}\right) \times N_{y}$ and $e_{y}=\left(e_{y}, 1_{y}\right)$. Omit Lemmas 1.1, 1.2, and 1.5. If $a=(g, j) \epsilon$ $s_{u}$, define $a^{\prime}=\left(e_{u}, j\right)$. Lemma 1.6 is now proved by a simple calculation. The modifications in Lemmas $1.8,1.9$, and 1.11 are clear from the statement of Theorem 1.13. These modifications arise since we do not have full associativity. If $a, b \in J_{u^{\prime}}, b^{a}=a b e_{u}=e_{u} \in E\left(I_{u}\right)$. If $a \in J_{u}$ and $B \in I_{u^{\prime}} a B=B$ and, hence, $B^{a}=B$.

Conversely, assume that $S$ is an upper generalized Schreier product of $H$ by $J$. (Upper) associativity is established as in the proof of The orem 1.12. Suppose $B \sim a \sim b$. Hence, $B^{a}=B$ and $b^{a} \in E(H)$ by hypothesis. Thus, $(A, a)$. $(B, b)=(A \circ B, b)$. Hence, if $A \in H_{y}, H_{y} \times J_{y}$ is a right group by [3, Theorem 
1.27]. Hence, $S$ is an upper associative semilattice $Y$ of right groups $\left(I_{y} \times J_{y}\right.$ : $y \in Y$ ). Q.E.D.

If $S$ is an upper (lower) associative semilattice $Y$ of semigroups $\left(S_{y}: y \in Y\right)$, a subset $T$ of $S$ is termed a partial subgroupoid of $S$ if $a \in T \cap S_{y}, b \in T \cap S_{z}$, and $y \leq z(y \geq z)$ imply $a b \in T$.

We next give a specialization of Theorem 1.13 which will have applications in $\$ 3$.

In the statement of Theorem 1.13 , let $J$ be an upper associative semilattice $Y$ of right zero semigroups, omit (3), delete all factor terms $b^{a}$, and impose "additional condition" if $a \in J, B \in E(I)$, and $a \leq B$, then $B^{a} \in E(I)$. We term the resulting structure an upper generalized semidirect product of $H$ by $J$.

Theorem 1.14. $S$ is an upper associative semilattice $Y$ of right groups sucb that $E(S)$ is a partial subgroupoid if and only if $S$ is upper isomorpbic to an upper generalized semidirect product of $H$ by $J$ for some $H$ and $J$.

Proof. Let $S$ be an upper associative semilattice $Y$ of right groups such that $E(S)$ is a partial subgroupoid. Let $J$ be as in the proof of Theorem 1.13. If $a, b \in J$ and $a \leq b, a * b=a b$ by virtue of Lemma 1.6. Hence, $(J, *)$ is upper associative. If $C \geq b \geq a,(a b) C=C^{a * b}(a * b)^{C}$ while $a(b C)=a\left(C^{b} b^{C}\right)=$ $\left(a C^{b}\right) b^{C}=\left(C^{b}\right)^{a}\left(a^{(\bar{C} b)} * b^{C}\right)$. Hence, $C^{a * b}=\left(C^{b}\right)^{a}$ and $(a * b)^{C}=a^{\left(C^{b}\right)} * b^{C}$. Thus, we obtain the modified form of (2) of Theorem 1.12. If $a \in J$ and $B \in E(I)$, and $a \leq B, a B \in E(S)$ since $E(S)$ is a partial subgroupoid. Hence, since $a B=$ $B^{a} a^{B}$ by Lemma $1.4, B^{a} \in E(I)$ by a simple calculation. If $a \leq b$ and $a \in J_{u}$, $b^{a}=a b e_{u}=e_{u}$. Hence, $b^{\left(a^{B}\right)}$ may be omitted in (4) of Theorem 1.12. Conversely, let $S$ be an upper generalized semidirect product of $H$ by $J$ for some $H$ and $J$. Using the modified forms of (1), (2), and (4) of Theorem 1.12, upper associativity is established by a simple calculation. As in the proof of Theorem 1.13, the condition $B^{a}=B$ if $a \sim B$ is used to prove $H_{y} \times J_{y}$ is a right group for each $y \in Y$. Clearly, $E\left(H_{y} \times J_{y}\right)=E\left(H_{y}\right) \times J_{y}$. Hence, using the "additional condition", $E(S)$ is a partial subgroupoid of $S$.

2. Bands of maximal left groups. In this section, we characterize a band of maximal left groups as a Schreier product of a lower associative semilattice $Y$ of left groups by an associative semilattice $Y$ of right zero semigroups.

We will need several lemmas.

A semigroup $X$ is termed a band $B$ of left groups if $X$ is the union of a collection of pairwise disjoint sub-left groups $\left(X_{b}: b \in B\right)$ where $B$ is a band (an idempotent semigroup) and $X_{u} X_{v} \subseteq X_{u v}$ for all $u, v \in B$. If, in addition, each $X_{b}(b \in B)$ is maximal in the collection of sub-left groups of $X, X$ is called a band $B$ of maximal left groups $\left(X_{b}: b \in B\right)$. 
The following lemma is an immediate consequence of [11, Lemma].

Lemma 21. A semigroup $S$ is a band of maximal left groups if and only if $S$ is a union of groups and $\mathfrak{\&}$ is a congruence on $S$.

In Lemmas 2.2-2.8, $S$ will be a band of maximal left groups.

Lemma 2.2. Let $A \in I_{s^{*}}$ Then, $b^{A}=b^{e s}$ for all $b \in \mathrm{J}$.

Proof. Since $\left(A, e_{s}\right) \in \mathscr{L},(b A, b e s) \in \mathscr{L}$ by Lemma 2.1. Thus, $\left(b^{A}, b^{e} s\right) \epsilon$ $\mathcal{Q}$ by Lemma 1.4, and hence $b^{A}=b^{e s}$.

Lemma 2.3. $(J, *)$ is an associative semilattice $Y$ of right zero semigroups $\left(J_{y}: y \in Y\right)$.

Proof. By virtue of Lemma 1.6, we must just establish associativity. In this proof $e_{v}$ is considered to be an element of $I_{v}$ for all $v \in Y$. Let $x \in J_{u}, y$ $\epsilon J_{v}$, and $z \in J_{w}$. Hence, $(x * y) * z=\left(x^{e} v y\right)^{\prime} * z=\left(\left(\left(x^{e} v y\right)^{\prime}\right)^{e} w_{z}\right)^{\prime}$ while $x *$ $(y * z)=x *\left(y^{e} w z\right)^{\prime}=\left(x^{e} v w\left(y^{e} w\right)^{\prime}\right)^{\prime}$. Utilizing Lemma 1.6, Lemma 1.9(a), Lemma 1.4, Lemma 2.2, and Lemma 1.8(a), $\left(\left(x^{e} v y\right)^{\prime}\right)^{e_{w}}=\left(x^{e} v * y\right)^{e_{w}}=\left(x^{e} v\right)^{(k)} * y^{e_{w}}=$ $\left(x^{e} v\right)^{e} v w * y^{e} w=x^{e} v w * y^{e} w=\left(x^{e} v w y^{e} w\right)^{\prime}$, where $k=e_{w}^{y}$. Hence, $\left(\left(\left(x^{e_{v}} y\right)^{\prime}\right)^{e_{w}}\right.$,

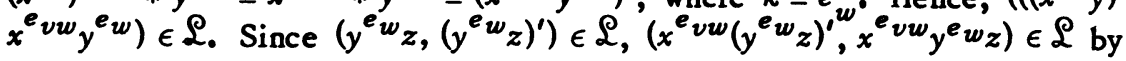

Lemma 2.1. Hence, $\left(\left(\left(x^{e} v y\right)^{\prime}\right)^{e} w_{z, x^{e} v w}\left(y^{e} w_{z}\right)^{\prime}\right) \in \mathfrak{L}$ and, thus, $(x * y) * z=x *$ $(y * z)$.

Lemma 2.4. If $j \in J, j^{e_{t}}=j * e_{t}$ (on the left consider $e_{t} \in I_{t}$ and on the right consider $\left.e_{t} \in J_{t}\right)$.

Proof. Since $j e_{t}=(j e) e_{t}, e_{i}^{j} j^{e}\left(e_{t} \in I_{t}\right)=\left(e_{t}^{j} j^{e}\right) e_{t}=e_{t}^{j}\left(j^{e} e_{t}\right)$ by Lemma 1.4. Hence, using Lemmas 1.4 and 1.2 and a simple calculation, $j^{e_{t}}=\left(j^{e_{t}} e_{t}\right)^{\prime}$. Thus, $j^{e_{t}}=j * e_{t^{\bullet}}$

Lemma 25. If $a \in J_{u}, b \in J_{v}, u \leq v$, and $C \in I_{w}, b^{a} \circ C^{a * b}=\left(C^{b}\right)^{a} \circ$ $\left(b * e_{w}\right)^{a * e_{v}} v w$.

Proof. Combine Lemmas 1.9(b), 2.2, and 2.4.

Lemma 26. If $A \in I_{u^{\prime}} B \in I_{v}, u \geq v$, and $c \in J$, then $(A \circ B)^{c}=A^{c} \circ B^{c * e_{u}}$.

Proof. We apply Lemmas 1.8(b), 2.2, and 2.4.

Lemma 27. If $a \in J_{u^{\prime}}, b \in J_{v^{\prime}}$ and $c \in J_{w^{\prime}}$ and $u \leq v \leq w, b^{a} \circ c^{a * b}=\left(c^{b}\right)^{a}$ $\circ(b * c)^{a * e}$.

Proof. Combine Lemmas 1.10(a), 2.2, and 2.4.

Lemma 2.8. $S \cong U\left(I_{y} \times J_{y}: y \in Y\right)$ under the multiplication 


$$
(A, a)(B, b)=\left(A \circ B^{a} \circ b^{\left(a * e_{t}\right)}, a * b\right)
$$

where $B \in I_{t}$.

Proof. We apply Lemmas 1.11, 2.2, 2.4, and 2.3. Q.E.D.

We are now in a position to state our structure theorem.

Let $(I, 0)$ be a lower associative semilattice $Y$ of left groups $\left(I_{y}: y \in Y\right)$ and let $(J, *)$ be an associative semilattice $Y$ of right zero semigroups $\left(J_{y}: y\right.$ $\epsilon Y)$ such that $I_{y} \cap J_{y}=\left(e_{y}\right)$, a single element, and let $H_{y}$ be the maximal subgroup of $I_{y}$ with identity $e_{y^{*}}$ For each $a \in J_{u}$ and $B \in I_{v}$, let $B^{a} \in I_{u v}$ and for each $a \in J_{u}$ and $b \in J_{v}$ with $u \leq v$ in $Y$, let $b^{a} \in H_{u}$. Assume the conditions:

1. If $A \geq B \in I$ with $A \in I_{u}$ and $c \in J,(A \circ B)^{c}=A^{c} \circ B^{c * e} u$.

2. If $a \leq b \in J$ with $b \in J_{v}$ and $C \in I_{w^{,}} b^{a} \circ C^{a * b}=\left(C^{b}\right)^{a} \circ\left(b * e e_{w}\right)^{a * e} v w$. 3. If $a \leq b \leq c \in J$ with $b \in J, b^{a} \circ c^{a * b}=\left(c^{b}\right)^{a} \circ(b * c)^{a * e v}$.

We term $U\left(I_{y} \times J_{y}: y \in Y\right)$ under the multiplication

$$
(A, a)(B, b)=\left(A \circ B^{a} \circ b^{\left(a * e_{t}\right)}, a * b\right)
$$

where $B \in I_{t}$ a Schreier product of $I$ by $J$.

Theorem 2.9. $S$ is a band of maximal left groups if and only if $S$ is a Schreier product of $I$ by $J$ for some $I$ and $J$.

Proof. Let $S$ be a band of maximal left groups. Hence, $S$ is a Schreier product of $I$ by $J$ by Lemmas $1.4,1.5,2.3$, the definition of $b^{a}$, and Lemmas $2.6,2.5,2.7$, and 2.8. To establish the converse, let $S$ be a Schreier product of $I$ by $J$. Let $a^{B}=a * e_{v}$ if $B \in I_{v}$ and, then, apply Theorem 1.12 to show $S$ is a semigroup. For $j \in J_{s}$, let $T_{j}=I_{s} \times(j)$. By the proof of Theorem 1.12, $T_{j}$ is a left group for each $j \in J_{s^{\circ}}$. Hence, using the multiplication given in the statement of Theorem 2.9, $S$ is a band $J$ of maximal left groups $\left(T_{j} ; j \in J\right)$.

Remark. In [11], we gave a structure theorem for bands of maximal left groups using another method of proof. The advantage of the present theorem is that it draws the factor terms $b^{a}$ from $\bigcup\left(H_{y}: y \in Y\right)$. The former theorem just limited the $b^{a}$ to $I$.

3. Orthogroups (orthodox unions of groups). If $S$ is an orthogroup in Theorem 1.12, $J$ is an upper associative semilattice of right zero semigroups and the factor terms $b^{a}$ may be deleted. We call the resulting structure a generalized semidirect product of $I$ by $J$. In this section, we characterize an orthogroup as a generalized semidirect product of $I$ by $J$. As a corollary to our structure theorem we give a variant of a previous structure theorem [10, Theorem 2] for bands (idempotent semigroups). 
As usual, we proceed by a sequence of lemmas. In Lemmas $3.1-3.6, S$ is an orthogroup.

Lemma 3.1. $(J, *)$ is an upper associative semilattice $Y$ of right zero semigroups $\left(J_{y}: y \in Y\right)$. If $a, b \in J$ and $a \leq b, a * b=a b$.

Proof. If $a, b \in J$ and $a \leq b, a * b=a b$ by Lemmas 1.2 and 1.6.

Lemma 3.2. If $a, b \in J$ witb $a \leq b$ and $a \in J_{u^{\prime}} b^{a}=e_{u^{*}}$

Proof. Using Lemma 3.1, $b^{a}=a b e_{u}=e_{u}$. Q.E.D.

Lemma 3.3. If $a \leq b \in J$ and $C \in I, C^{a * b}=\left(C^{b}\right)^{a}$.

Proof. Let $a, b \in J$ with $a \leq b$ and let $C \in I$. By Lemmas 3.2 and 3.1, $b^{a}(a * b)=a * b$, and, hence, $b^{a}((a * b) C)=(a * b) C$. Thus, $b^{a}\left(C^{a * b}(a * b)^{c}\right)=$ $C^{a * b}(a * b)^{C}$ by Lemma 1.4. Thus, using Lemmas 1.3 and $1.5, C^{a * b}=b^{a} \circ C^{a * b}$. We then apply Lemmas 1.9(b) and 3.2.

Lemma 3.4. If $A \in I, a \in J$, and $A \sim a, A^{a} \in E(S)$ if and only if $A \in E(S)$.

Proof. The proof is by a simple calculation.

Lemma 3.5. $S \cong \bigcup\left(I_{y} \times J_{y}: y \in Y\right)$ under the multiplication $(A, a)(B, b)=$ $\left(A \circ B^{a}, a^{B} * b\right)$.

Proof. We apply Lemmas 3.2 and 1.11. Q.E.D.

We are now in a position to state our structure theorem for orthogroups.

Let $(I, 0)$ be a lower associative semilattice $Y$ of left groups $\left(I_{y}: y \in Y\right)$ and let $(J, *)$ be an upper associative se milattice $Y$ of right zero semigroups $\left(J_{y}: y \in Y\right)$. For each $a \in J_{u}$ and $B \in I_{v}(u, v \in Y)$, let $B^{a} \in I_{u v}$ and $a^{B} \in J_{u v}$. Assume the conditions:

1. If $A \geq B \in I$ and $c \in J$,

(a) $c^{A{ }^{\circ}}=\left(c^{A}\right)^{B}$,

(b) $(A \circ B)^{c}=A^{c} \circ B^{\left(c^{A}\right)}$.

2. If $a \leq b \in J, C \in I$,

(a) $C^{a * \bar{b}}=\left(C^{b}\right)^{a}$,

(b) $(a * b)^{C}=a^{\left(\dot{C}^{b}\right)} * b^{C}$.

3. If $A \sim a, A^{a} \in E(I)$ if and only if $A \in E(I)$.

We term $U\left(I_{y} \times J_{y}: y \in Y\right)$ under the multiplication $(A, a)(B, b)=\left(A \circ B^{a}\right.$, $\left.a^{B} * b\right)$ a generalized semidirect product of $I$ by $J$.

Theorem 3.6. $S$ is an ortbogroup if and only if $S$ is a generalized semidirect product of $I$ by $J$ for some $I$ and $J$. 
Proof. Let $S$ be an orthogroup. Hence, $S$ is a generalized semidirect product of $I$ by $J$ by Lemmas 1.5, 3.1, 1.4, 3.3, 1.8, 1.9(a), 3.4, and 3.5. Conversely, let $S$ be a generalized semidirect product of $I$ by $J$. Using (1) and (2), it is easily established that $S$ is a semigroup. Just as in the proof of Theorem 1.12, it is shown that $T_{s}$ (notation of Theorem 1.12) is a completely simple semigroup. Using (3), $E\left(T_{s}\right)=\left((A, a): A \in E\left(I_{s}\right)\right)$. Using (3) and the fact that $E\left(I_{s}\right)$ is a semigroup, $E\left(T_{s}\right)$ is a semigroup. Hence, $T_{s}$ is a rectangular group (the algebraic direct product of a group and a rectangular band). Thus, $S$ is the semilattice $Y$ of rectangular groups $\left(T_{y}: y \in Y\right)$. Hence, by a result of Preston, Yamada, and Clifford [4, Proposition 1], $S$ is an orthogroup.

Remark 3.7. We will show that $E(I)$ in Theorem 3.6 is a partial subgroupoid of $I$. Hence, the dual of Theorem 1.14 may be used to further refine the structure of $I$. Using (3), $(A, a) \in E(S)$ if and only if $A \in E(I)$. Let $b \in J_{t^{\prime}} A \in E\left(I_{s}\right)$, $B \in E\left(I_{t}\right)$, and $t \leq s$. Hence, $A^{b} \in I_{t}$. Since $I_{t}$ is a left group, there exists $C \epsilon$ $E\left(I_{t}\right)$ such that $\bar{C} \circ A^{b}=A^{b}$. Thus, if $a \in J_{s},(C, b)(A, a)=\left(A^{b}, b^{A} * a\right) \in E(S)$, and, hence $A^{b} \in E\left(I_{t}\right)$. Thus, using (1) and (3), $(A \circ B)^{b}=A^{b} \circ B^{\left(b^{A}\right)}=A^{b}$, and $A \circ B \in E\left(I_{t}\right)$ by (3).

Remark. For other characterizations of orthogroups see the papers of Clifford [4] and M. Yamada [12].

Let $(I, 0)$ be a lower associative semilattice $Y$ of left zero semigroups $\left(I_{y}\right.$ : $y \in Y$ ) and omit (3) in the statement of Theorem 3.6. We term the resulting structure a generalized semidirect product of $E(I)$ by $J$.

Corollary 3.8 (cf. Warne [10]). $S$ is an idempotent semigroup if and only if $S$ is a generalized semidirect product of $E(I)$ by $J$ for some $E(I)$ and $J$.

Remark. The referee points out that a structure theorem for idempotent semigroups has been also given by M. Petrich in [G].

4. Generalized $R$-unipotent unions of groups. A generalized $R$-unipotent semigroup is a regular semigroup whose set of idempotents $E$ obey the condition: $e, f \in E$ and $e f=f$ imply that $f e g f g=f g$ for $g \in E$. We studied the structure of such semigroups in [9]. By [9, Lemma 1], a regular semigroup is a generalized R-unipotent semigroup if and only if $E$ is a semigroup and $R$ is a congruence on $E$. In this section, we characterize a generalized $R$-unipotent union of groups as a semidirect product of $J$ by $I$. Here, $I$ is an associative semilattice $Y$ of left groups and $J$ is an upper associative semilattice $Y$ of right zero semigroups.

We will need the following definitions. Let $S$ be a lower associative semilattice $Y$ of groupoids $\left(S_{y}: y \in Y\right)$ and let $X$ be a semigroup. A mapping $\gamma$ of $S$ into $X$ is termed a lower homomorphism of $S$ into $X$ if $r, s \in S$ and $r \geq s$ imply $(r s) y=r \gamma s y$. 
For $A \in I$ and $b \in J$, set $A a_{b}=A^{b}$ and $b \theta_{A}=b^{A}$.

In Lemmas 4.1-4.6, $S$ is a generalized R-unipotent union of groups.

Lemma 4.1. $(e, f) \in R \in E(S) \times E(S)$ and $p \in S$ imply $(e p, f p) \in R$.

Proof. Suppose $(e, f) \in R \cap E(S) \times E(S)$. Hence, for $p \in S,\left(e p p^{-1}, f p p^{-1}\right) \epsilon$ $R\left(p^{-1}\right.$ is the group inverse of $p$ in the group containing $\left.p\right)$. Thus, $e p p^{-1} f p p^{-1}$ $=f p p^{-1}$ and $f p p^{-1} e p p^{-1}=e p p^{-1}$. Hence, $e p\left(p^{-1} f p\right)=f p$ and $f p\left(p^{-1} e p\right)=e p$ and, thus, $(e p, f p) \in R$.

Lemma 4.2. If $j \in J_{s^{\prime}} a_{j}=a_{e_{s}}$.

Proof. Let $j=\left(e_{s}, 1_{s}, j\right)$ where $j \in N_{s}$ and $A=\left(g, i, 1_{t}\right)$ where $g \in G_{t}$ and $i \in M_{t}$. Hence, using Lemma 3.1, $e_{s t} j=\left(e_{s t}, 1_{s t}, j^{\prime}\right)$ for some $j^{\prime} \in N_{s t}$ and, similarly, $e_{s t} e_{s}=\left(e_{s t}, 1_{s t}, k\right)$ for some $k \in N_{s t^{*}}$. However, $A e_{s t}=\left(g^{g}, a, 1_{s t}\right)$ for some $g^{\prime} \in G_{s t}$ and $a \in M_{s t}$ by Lemma 1.1. Hence, $e_{s t} j A e_{s t}=\left(g^{\prime}, 1_{s t}, 1_{s t}\right)=$ $e_{s t} e_{s} A e_{s t^{\circ}}$ Next, suppose that $j A=(w, m, n) \in S_{s t}$ and $e_{s} A=(u, c, d) \in S_{s t^{\circ}}$ Hence, $e_{s t} j A e_{s t}=\left(w, 1_{s t}, 1_{s t}\right)$ and $e_{s t} e_{s}^{A e_{s t}}=\left(u, 1_{s t}, 1_{s t}\right)$ and, thus, $w=u$. By Lemma 4.1, $\left(j, e_{s}\right) \in \mathscr{R}$ implies $\left(j A, e_{s} A\right) \in R$. Thus, $j A=(w, m, n)=$ $\left(w, m, 1_{s t}\right)\left(e_{s t}, 1_{s t}, n\right)$ while $e_{s} A=(w, m, d)=\left(w, m, 1_{s t}\right)\left(e_{s t}, 1_{s t}, d\right)$. Hence, $A a_{j}=$ $A a_{e_{s}}$

Lemma 4.3. $(I, 0)$ is an associative semilattice $Y$ of left groups $\left(I_{y}: y \in Y\right)$.

Proof. By Lemma 1.5, we must just establish associativity. Let $A \in I_{s}, B$ $\epsilon I_{y}$, and $C \in I_{t^{\circ}}$ Utilizing Lemma 1.5, $(A \circ B) \circ C=\left(A\left(B a_{e_{s}}\right)\right) \circ C=A\left(B a_{e_{s}}\right) C a_{e_{s y}}$ while $A \circ(B \circ C)=A \circ\left(B\left(C a_{e_{y}}\right)\right)=A\left(\left(B\left(C a_{e_{y}}\right)\right) a_{e_{s}}\right)$. We apply Lemmas $1.8(\mathrm{~b})$, 4.2, and 3.3.

Lemma 4.4. If $A \in I, A a_{e_{v}}=e_{v} \circ A$.

Proof. Since $e_{v}\left(e_{v} A\right)=e_{v} A,\left(e_{v}\left(A a_{e_{v}}\right)\right) e_{v} \theta_{A}=A a_{e_{v}} e_{\nu} \theta_{A}$ by Lemma 1.4. Hence, utilizing Lemma 1.3 and the definition of " $\sigma$ ", $A a_{e_{v}}=e_{v}\left(A a_{e_{v}}\right)=e_{v} \circ A$.

Lemma 4.5. If $a \in J_{u^{,}} b \in J_{v^{,}} u \leq v$, and $C \in I,(a * b) \theta_{C}=a \theta_{e_{v}}{ }{ }^{*} b \theta_{C^{\circ}}$

Proof. By Lemmas 4.2 and 4.4, $C a_{b}=C a_{e_{v}}=e_{v} \circ C$. Apply Lemma 1.9(a).

Lemma 4.6. $S \cong \bigcup\left(I_{y} \times J_{y}: y \in Y\right)$ under the multiplication $(A, a)(B, b)=$ $\left(A \circ B, a \theta_{B} * b\right)$.

Proof. Combine Lemmas 3.5, 4.2, 4.4, and 4.3. Q.E.D.

If $X$ is a set, $T_{X}$ will denote the full transformation semigroup on $X$. 
We are now in a position to state our structure theorem.

Let $(I, 0)$ be an associative semilattice $Y$ of left groups $\left(I_{y}: y \in Y\right)$ and let $(J, *)$ be an upper associative semilattice $Y$ of right zero semigroups $\left(J_{y}\right.$ : $y \in Y$ ) such that $I_{y} \cap J_{y}=\left(e_{y}\right)$, a single element. Let $B \rightarrow \theta_{B}$ be a lower homomorphism of $(I, 0)$ into $T_{J}$ subject to the condition:

1. If $C \in I_{u}, J_{y} \theta_{C} \subseteq J_{u y}$.

2. If $C \in I, b \in J_{v}$, and $a \leq b$ (in J), $(a * b) \theta_{C}=a \theta_{e_{v} \propto C} * b \theta_{C}$.

We term $U\left(I_{y} \times J_{y}: y \in Y\right)$ under the multiplication $(A, a)(B, b)=(A \circ B$, $\left.a \theta_{B} * b\right)$, a semidirect product of $J$ by $I$.

Theorem 4.7. $S$ is a generalized R-unipotent union of groups if and only if $S$ is a semidirect product of $J$ by $I$ for some $J$ and $I$.

Proof. Let $S$ be a generalized R-unipotent union of groups. Hence, $S$ is a semidirect product of $J$ by $I$ by Lemmas $4.3,3.1,1.8(a), 1.4,4.5$, and 4.6. To establish the converse, let $S$ be a semidirect product of $J$ by $I$. If $A \in I$, let $A a_{b}=e_{v} \circ A$ if $b \in J_{v}$, and then apply Theorem 3.6 to show that $S$ is an orthogroup. By the proof of Theorem 3.6, $E(S)=((A, a): A \in E(I))$. By a simple calculation, $(A, a) R(B, b)(\epsilon E(S))$ if and only if $A=B$. Hence, $R$ is a congruence on $E(S)$, and, thus, $S$ is generalized $R$-unipotent.

5. Orthodox bands of maximal left groups. We combine results of $\$ \$ 2$ and 3 to show that $S$ is a band of maximal left groups such that $E(S)$ is a semigroup ( $S$ is an orthodox band of maximal left groups) if and only if $S$ is a semidirect product of $I$ by $J$. Here, $I$ is a lower associative semilattice $Y$ of left groups and $J$ is an associative semilattice $Y$ of right zero semigroups. As a corollary, we give a variant of a previous structure theorem [10, Theorem 3$]$ for bands of maximal left zero semigroups.

We will need the following definition. Let $S$ be an upper associative semilattice $Y$ of groupoids $\left(s_{y}: y \in Y\right)$. A mapping $\gamma$ of $S$ into $X$ is termed an upper antihomomorphism of $S$ into $X$ if $r, s \in S$ and $r \leq s$ imply (rs)y $=s \gamma r y$.

We now state our structure theorem.

Let $(I, 0)$ be a lower associative semilattice $Y$ of left groups $\left(I_{y}: y \in Y\right)$, let $(J, *)$ be an associative semilattice $Y$ of right zero semigroups $\left(J_{y}: y \in Y\right)$ such that $I_{y} \cap J_{y}=\left(e_{y}\right)$, a single element, and let $a \rightarrow a_{a}$ be an upper antihomomorphisin of $(J, *)$ into $T_{I}$ subject to the conditions:

1. If $r \in J_{v}, I_{y} a_{r} \subseteq I_{y v}$.

2. If $B \leq A, A \in I_{u}$, and $c \in J,(A \circ B) a_{c}=A a_{c} \circ B a_{c * e_{u}}$.

3. If $A \sim a, A a_{a} \in E(I)$ if and only if $A \in E(I)$.

We term $\bigcup\left(I, \times J_{y}: y \in Y\right)$ under the multiplication $(A, a)(B, b)=\left(A \circ B a_{a}\right.$, $a * b)$, a semidirect product of $I$ by $J$.

Theorem 5.1. $S$ is an ortbodox band of maximal left groups if and only if $S$ is a semidirect product of $I$ by $J$ for some $I$ and $J$. 
Proof. Let $S$ be an orthodox band of maximal left groups. Hence, $S$ is a semdirect product of $I$ by $J$ by Lemmas $1.5,2.3,3.3,1.4,1.8(\mathrm{~b}), 2.2,2.4,3.4$, and 3.5. Conversely, let $S$ be a semidirect product of $I$ by $J$. Define $a \theta_{B}=a * e_{u}$ if $B \in I_{u}$. Then, by an application of Theorem 3.6, $S$ is an orthogroup. Thus, if $T_{j}=I_{s} \times(j)$ for $j \in J_{s}, S$ is the band $J$ of maximal left groups $\left(T_{j}: j \in J\right)$.

Let $(I, 0)$ be a lower associative semilattice $Y$ of left zero semigroups $\left(I_{y}\right.$ : $y \in Y$ ) and omit (3) in the statement of Theorem 5.1. We term the resulting structure a semidirect product of $E(l)$ by $J$.

Corollary 5.2 (cf. Warne [10]). $S$ is a band of maximal left zero semigroups if and only if $S$ is a semidirect product of $E(I)$ by $J$ for some $E(I)$ and $J$.

6. Generalized R-unipotent bands of maximal left groups. Let $(I, 0)$ be an associative semilattice $Y$ of left groups $\left(I_{y}: y \in Y\right)$ and let $(J, *)$ be an associative semilattice $Y$ of right zero semigroups $\left(J_{y}: y \in Y\right)$. Denote $U\left(I_{y} \times J_{y}: y \in\right.$ $Y)$ under the multiplication $(A, a)(B, b)=(A \circ B, a * b)$ by $I \times_{Y} J$.

Theorem 6.1. $S$ is a generalized R-unipotent band of maximal left groups if and only if $S \cong I \times_{Y} J$ for some $I$ and $J$.

Proof. Let $S$ be a generalized $R$-unipotent band of maximal left groups. Hence, $S \cong I \times_{Y} J$ by Theorem 5.1 and Lemmas 4.2, 4.3, and 4.4. The converse is valid by a direct calculation. Q.E.D.

Remark 6.2 In the terminology of Kimura [s], $I \times_{Y} J$ would be termed the spined product of $I$ and $J$ with respect to $Y$. The notation $I \times_{Y} J$ is due to Clifford [4].

We now specialize Theorem 6.1 to idempotent semigroups to obtain a slight variant of a theorem of Kimura [5, Theorem 2].

Corollary 6.2. $S$ is a band on which $R$ and $\mathcal{L}$ are congruences if and only if $S$ is the spined product of an associative semilattice of left zero semigroups and an associative semilattice of right zero semigroups.

7. $\mathcal{L}$-unipotent unions of groups. A semigroup $S$ is termed $\mathcal{Q}$-unipotent if each $\mathcal{L}$-class of $S$ contains precisely one idempotent. We studied the structure of such semigroups in [8]. Using the discussion at the beginning of $\S 1, S$ is an $\mathcal{L}$-unipotent union of groups if and only if $S$ is an associative semilattice $Y$ of right groups $\left(S_{y}: y \in Y\right)$. We show that $S$ is an ${ }_{\text {-unipotent union of groups if }}$ and only if $S$ is a semidirect product of $J$, an associative semilattice $Y$ of right zero semigroups $\left(J_{y}: y \in Y\right)$, by $I$, an associative semilattice $Y$ of groups $\left(I_{y}\right.$ : $y \in Y$ ). (Thus, the structure of $I$ is known mod groups and homomorphisms by a well-known result of Clifford ([2], [3, Theorem 4.11]) (Theorem 7.2).)

As a corollary to The orem 7.2, we obtain Bailes' theorem that $S$ is an $\mathcal{L}$. unipotent band of groups if and only if $S \cong I \times{ }_{Y} J$. 


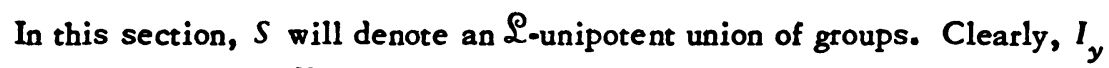
is a group for each $y \in Y$.

Lemma 7.1. $(J, *)$ is an associative semilattice $Y$ of right zero semigroups $\left(J_{y}: y \in Y\right)$.

Proof. Let $x \in J_{u} y \in J_{v}$, and $z \in J_{w}$. Utilizing Lemmas 4.5, 3.1, 4.3, and 1.8(a), $(x * y) * z=\left(x \theta_{e_{v}} y\right) * z=\left(x \theta_{e_{v}} y\right) \theta_{e_{w}} z=\left(x \theta_{e_{v}} \theta_{e_{v} e_{w}} y \theta_{e_{w}}\right) z=$ $\left(x \theta_{e_{v}{ }^{e_{w}}} y \theta_{e_{w}}\right) z=\left(x \theta_{e_{v w}} y \theta_{e_{w}}\right) z=x \theta_{e_{v w}}\left(y \theta_{e_{w}} z\right)=x *\left(y \theta_{e_{w}} z\right)=x *(y * z)$.

In the next theorem, we use the definition of semidirect product given in the statement of Theorem 4.7.

Theorem 7.2. $S$ is an $\mathcal{L}$-unipotent union of groups if and only if $S$ is a semidirect product of $(J, *)$, an associative semilattice $Y$ of right zero semigroups $\left(J_{y}: y \in Y\right)$, by $(I, 0)$, an associative semilattice $Y$ of groups $\left(I_{y}: y \in Y\right)$.

Proof. Let $S$ be an $\mathcal{L}$-unipotent union of groups. Hence, $S$ is a semidirect product of $J$ by $I$ (the $J$ and $I$ given in the statement of the theorem) by Lemmas 4.3, 7.1, 1.8(a), 1.4, 4.5, and 4.6. Conversely, let $S$ be a semidirect product of $J$ by $I$. Hence, $S$ is a generalized R-unipotent union of groups by Theorem 4.7. By a simple calculation, $E(S)$ is the semilattice $Y$ of right zero semigroups $\left(\left(\left(e_{s}\right) \times J_{s}\right): s \in Y\right)$. Hence, $S$ is $\mathcal{L}$-unipotent by [8, Proposition 5].

Corollary 7.3 (Bailes), $S$ is an $\mathcal{L}$-unipotent band of groups if and only if $S$ is the spined product of an associative semilattice of groups and an associative semilattice of right zero semigroups.

Proof. Let $S$ be an $\mathcal{Q}$-unipotent band of groups. Hence, $S$ is a generalized $R$-unipotent band of maximal left groups. Thus, by Theorem $6.1, S \cong I \times_{Y} J$ where $I$ is an associative semilattice $Y$ of left groups $\left(I_{y}: y \in Y\right)$ and $J$ is an associative semilattice $Y$ of right zero semigroups $\left(J_{y}: y \in Y\right)$. Since $S$ is \&-unipotent, $I_{y}$ is a group for each $y \in Y$. Conversely, let $S=I \times_{Y} J$ where $I$ and $J$ are as in the statement of Corollary 7.3. Let $H_{j}=I_{y} \times(j)$ for $j \in J_{y}$. Hence, $S$ is the band $J$ of groups $\left(H_{j}: j \in J\right), S$ is $\mathcal{L}$-unipotent as in the proof of Theorem 7.2.

Remark. The referee points out that Bailes theorem is also a special case of a result of $M$. Yamada [12].

\section{REFERENCES}

1. Gordon L. Bailes, Right inverse semigroups, Semigroup Forum (to appear).

2. A. H. Clifford, Semigroups admitting relative inverses, Ann. of Math (2) 42 (1941), 1037-1049. MR 3, 199.

3. A. H. Clifford and G. B. Preston, The algebraic the ory of semigroups. Vol. I, Math. Surveys, no. 7, Amer. Math. Soc., Providence, R. I., 1961. MR 24 \#A2627. 
4. A. H. Clifford, The structure of orthodox unions of groups, Semigroup Forum 3 (1972), 283-337.

5. Naoki Kimura, Note on idempotent semigroups. I, Proc. Japan Acad. 33 (1957), 642-645. MR $20 \# 4602$.

6. M. Petrich, Topics in semigroups, Lecture Notes, Pennsylvania State University, 1967, 534 pp.

7. D. Rees, On semigroups, Proc. Cambridge Philos. Soc. 36 (1940), 387-400. MR 2, 127.

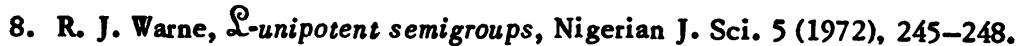

9. Generalized \&-unipotent semigroups, Bol. Un. Mat. Ital. 5 (1972), 43-47.

10. On the structure of idempotent semigroups, Proc. Amer. Math. Soc. 37 (1973), 17-21.

11. - Bands of maximal left groups, Rev. Roumaine Math. Pures Appl. 27 (1972), $1705-1707$.

12. M. Yamada, Strictly inversive semigroups, Bull. Shimane Univ. Nat. Sci. 13 (1964), $128-138$.

13. Construction of inversive semigroups, Mem. Fac. Lit. Sci. Shimane Univ. Natur. Sci. 4 (1971), 1-9.

DEPARTMENT OF MATHEMATICS, UNIVERSITY OF AL ABAMA, BIRMINGH AM AL ABAMA 35233 